\title{
Precision Gravity Tests with Atom Interferometry in Space
}

\author{
G. M. Tino ${ }^{\mathrm{a}}$, F. Sorrentino ${ }^{\mathrm{a}}$, D. Aguilera ${ }^{\mathrm{b}}$, B. Battelier ${ }^{\mathrm{c}}$, A. Bertoldi ${ }^{\mathrm{c}}$, Q. Bodart $^{\mathrm{a}}$, K. Bongs $^{\mathrm{d}}$, P. Bouyer $^{\mathrm{c}}$, C.

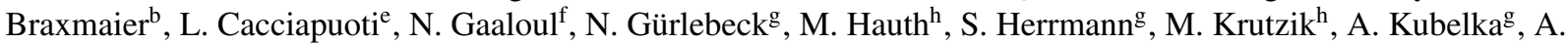

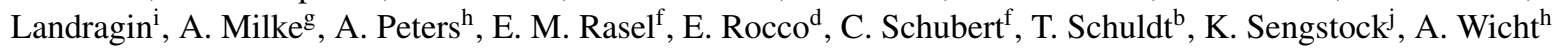 \\ ${ }^{a}$ Dipartimento di Fisica e Astronomia $\mathcal{E}$ LENS, Università di Firenze, INFN Sezione di Firenze, via Sansone 1, I-50019 Sesto Fiorentino, Italy \\ ${ }^{b}$ Institute of Space Systems, German Aerospace Center, Robert-Hooke-Strasse 7, 28359 Bremen, Germany \\ ${ }^{c}$ Laboratoire Photonique, Numérique et Nanosciences, LP2N - UMR5298 - IOGS - CNRS Université Bordeaux 1, Bâtiment A30 351 cours de la \\ Libération F- 33405 TALENCE Cedex France \\ ${ }^{d}$ Midlands Ultracold Atom Research Centre School of Physics $\mathcal{E}$ Astronomy University of Birmingham, Edgbaston, Birmingham B15 2TT, UK \\ ${ }^{e}$ European Space Agency, Research and Scientific Support Department, Keplerlaan 1, 2201 AZ Noordwijk, The Netherlands \\ ${ }^{f}$ Institute of Quantum Optics, Leibniz Universitaet Hannover, Welfengarten 1, D 30167 Hannover, Germany \\ ${ }^{g}$ University of Bremen, Centre of Applied Space Technology and Microgravity (ZARM), Am Fallturm, D - 29359 Bremen, Germany \\ ${ }^{h}$ Humboldt-Universität zu Berlin, Newtonstr. 15, D-12489 Berlin, Germany \\ ${ }^{i}$ LNE-SYRTE, Observatoire de Paris, CNRS, UPMC and LNE, 61 avenue de l'Observatoire 75014 Paris, France \\ ${ }^{j}$ Universität Hamburg, Edmund-Siemers-Allee 1, D-20146 Hamburg, Germany
}

\begin{abstract}
Atom interferometry provides extremely sensitive and accurate tools for the measurement of inertial forces. Operation of atom interferometers in microgravity is expected to enhance the performance of such sensors. This paper presents two possible implementations of a dual ${ }^{85} \mathrm{Rb}-{ }^{87} \mathrm{Rb}$ atom interferometer to perform differential gravity measurements in space, with the primary goal to test the Weak Equivalence Principle. The proposed scheme is in the framework of two projects of the European Space Agency, namely Q-WEP and STE-QUEST. The paper describes the baseline experimental configuration, and discusses the technology readiness, noise and error budget for the two proposed experiments.
\end{abstract}

Keywords: Atom interferometry, Gravity measurements in space, Weak Equivalence Principle

\section{Atom Interferometers and WEP tests}

Matter-wave interferometry has recently led to the development of new techniques for the measurement of inertial forces, with important applications both in fundamental physics and applied research. The remarkable stability and accuracy that atom interferometers (AI) have reached for acceleration measurements can play a crucial role for science and technology. Quantum sensors based on atom interferometry had a rapid development during the last decade and different measurement schemes were demonstrated and implemented. Gravity measurements using atom interferometry are being performed with ever increasing precision. Atom interferometers have been applied in gravitational physics, proving alternative measurements of $\mathrm{G}$ with substantially different systematic effects $[1,2]$ and more generally competitive gravimetric measurements [3]. Peters et al. [4] have been able to measure the local gravitational acceleration by dropping cold atoms with an absolute uncertainty of $3 \cdot 10^{-9}$, and this is the best result so far. A comparison with an absolute gravimeter based on falling corner cube, with a quoted relative uncertainty of 2 ppb showed a difference of $7 \pm 7$ ppb due to the uncertainty of the gravity gradient measurement of $5 \mathrm{ppb}$. The resolution of the atom interferometer has been about four times higher than the FG- 5 due to the higher repetition rate, the noise behaviour was similar. Gravity gradiometers have been realized with a pair of simultaneous atom interferometers, vertically displaced 
by a few tens of $\mathrm{cm}$. This kind of sensor measures the gradient of gravity $\nabla g$ with a precision approaching state-of-the-art mobile gravity-gradient sensors $[5,6]$.

Since these pioneering experiments, AI sensors have been further developed for special applications and transportable sensors. Most prominent examples are the atomic sensors developed by M. Kasevich at Stanford University or the gravi-gradiometer at the JPL by N. $\mathrm{Yu}$ in the US as well as current developments at the SYRTE (PARIS), the LENS (Florence), Leibniz Universität Hannover, Humboldt Universität zu Berlin, and Birmingham University. These activities comprise sensors to measure the Newtonian constant, terrestrial gravity and the Earth rotation rate.

Atom interferometers can also be exploited to test general relativity [7]. A Local Lorentz Invariance test has been performed by Mueller et al. [8]. Proposals also exist to exploit atom interferometry to test for deviations from the Newtonian inverse square law at short distances $[9,10]$.

The equivalence of gravitational and inertial mass is, together with Local Position Invariance, the Universality of the Gravitational Redshift and Local Lorentz Invariance, one of the cornerstones of general relativity. This equivalence results in the Universality of Free Fall: free fall is independent of test mass composition. The free fall universality is investigated with classical (bulk) matter e.g. in the lunar laser ranging [11] and torsion balance [12] experiments and no deviation has been shown in parts of $10^{-13}$. Tracking the free propagation of matter waves extends free fall experiments in the domain of quantum objects. We consider it as a conceptually different approach compared to all other free-fall tests based on classical bodies. According to quantum mechanics, particles have to be described as wave packets which implies the concept of coherence of the different partial waves. Current debates on the interpretation of the measurements performed with matter waves emphasize these conceptual differences. Its results can be interpreted as tests of General Relativity and other metric theories of gravity and as tests for the existence of new fields associated to matter.

An atom interferometry test of WEP is based on the simultaneous acceleration measurement of two different atomic species, or two different isotopes, in free fall. Fray et al. have already performed a proo-of-principle UFF test with sequential acceleration measurement on $\mathrm{Rb}$ isotopes, reaching $10^{-7}$ [13]. By using the same atom optics tool to manipulate the wave-packet of the two atomic species, a dramatic common-mode suppression of the main noise sources in the differential acceleration measurement is achieved. Experiments of this type in preparation at Stanford aim at measuring the relative gravitational acceleration of falling cold atoms ${ }^{85} \mathrm{Rb}$ and ${ }^{87} \mathrm{Rb}$ to $\sim 10^{-15}$ [7].

\section{WEP test with Atom Interferometry in space: Q- WEP and STE-QUEST ATI}

The sensitivity of atom interferometers to inertial accelerations scales as the square of the time in free fall. Ground based atom interferometric experiments are fundamentally limited, since an increasing time in free fall also means an increasing pathlength of the atom trajectory, making the control of systematics extremely challenging. An attractive alternative is the operation of an atom interferometer in a microgravity environment. Free-fall conditions allow to operate the instrument over long interrogation times still keeping a compact set up, crucial for ensuring adequate control on the environmental effects that could perturb the atom interferometry measurement (magnetic, thermal, etc.). The long interaction times achievable in space promise sensitivities to differential accelerations in the $10^{-12} \mathrm{~g}$ regime at about $1 \mathrm{~s}$ of integration time, compatible with a test of the Weak Equivalence Principle on quantum objects at the $10^{-15}$ level. Several developments, within The European Space Agency (ESA) and the national agengies CNES and DLR, are today investigating the potential of cold atom interferometry for precision measurements and fundamental tests in space. A compact cold atom inteferometry sensor has been developed within the ESA project SAI [14]. The sensor design, compatible with the Bremen drop-tower capsule, is studying key atom interferometry techniques as a first step for the space qualification of this technology. Within the European programme "Quantum Gases in Microgravity", intensive activities are carried on, financed on the national level, to bring instruments based on atom interferometry to maturity for space: parabolic flight experiments are ongoing in the CNES project ICE [15]; drop tower tests conducted by DLR in the QUANTUS project have demonstrated the robustness of miniaturised ultra-cold atom technology. The QUANTUS set-up is indeed able to routinely produce Bose Einstein Condensates (BEC) during the about $100 \mathrm{~m}$ of free fall in the Bremen drop tower and survive decelerations as high as $50 \mathrm{~g}$ [16]. The experiment also proved atomic interferometry with free evolution times in excess of $700 \mathrm{~ms}$ [17]. The sounding rocket mission MAIUS (also DLR funded) will be a test platform for a sounding rocket flight in order to demonstrate key elements of future long-duration space missions. The rocket will be equipped with an 
atom laser operated in an extended experimental parameters range compared to the drop tower. The MAIUS facility will carry a degenerate atom source for a total experiment time of $6 \mathrm{~min}$. The experiment will explore the necessary techniques to automate an atom interferometry experiment of several cycles without human intervention.

An atom interferometry sensor would strongly benefit from the technology development of the microwave clock PHARAO, developed by CNES in the frame of the ESA mission "Atomic Clock Ensemble in Space" (ACES): lasers and optical bench, atomic source, low phase noise microwave generation. It is expected that the use of degenerate quantum gases as sources for atom interferometry, combining the progress made with SAI, ICE and QUANTUS, will provide unique sensor capabilities for investigations in fundamental physics.

The International Space Station (ISS) is a platform which is specifically designed for experiments in a continuously available microgravity environment. In 2011 ESA launched the invitation to tender $\mathrm{AO} / 1$ 6763/11/NL/AF - Atom Interferometry Test Of the Weak Equivalence Principle in Space (Q-WEP) [18]. Mission background underlying the Q-WEP study is an atom interferometer which is adapted for operation on the ISS, with the main scientific objective to test the WEP. Additional applications like gravity gradiometry are assessed as well. The development and the space validation of cold atom interferometry sensors would make available new instruments for inertial measurements with an extremely good long-term stability and with a well known calibration factor. Such technology could find interesting applications in inertial navigation and geodesy.

STE-QUEST (Space Time Explorer and Quantum Equivalence Space Test) is an M-class mission candidate for the 2022/2024 launch slot in the Cosmic Vision M3 programme. STE-QUEST was recommended by the Space Science Advisory Committee (SSAC) to be studied first internally and afterwards with parallel industrial contracts. The proposed mission is devoted to a precise measurement of the effect of gravity on time and matter using an atomic clock (the STE-QUEST AC) and an atom interferometer (the STE-QUEST ATI). The mission will have two primary goals: to measure the Earth gravitational red shift with an accuracy of $10^{-7}$, making use of a highly elliptic orbit and advanced atomic clocks (see Fig. 1); and to perform a quantum test of the universality of free-fall by interferometrically tracking the propagation of matter waves in the Earth field, using two atomic samples of ${ }^{85} \mathrm{Rb}$ and ${ }^{87} \mathrm{Rb}$ and striving for an accuracy at the $10^{-15}$ level.

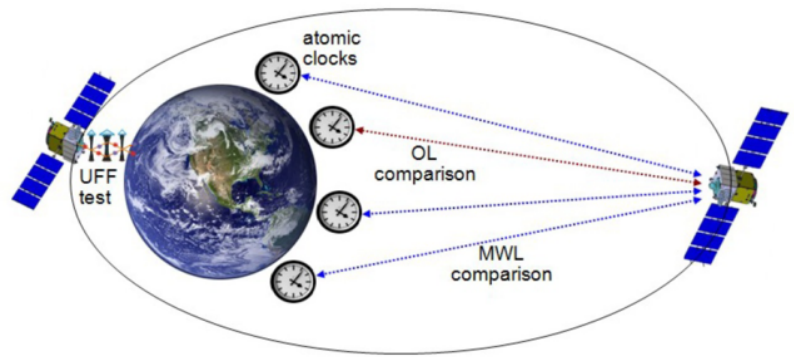

Figure 1: General concept of the STE-QUEST mission. The clock on the satellite is compared with one or more ground clocks as the satellite orbits earth on a highly elliptic orbit. During the perigee the local acceleration of two rubidium isotopes is measured and compared.

The baseline design of the atom interferometry payload, the experimental scheme and the nature of performance limits are similar for Q-WEP and for the STEQUEST ATI. In the following sections, a general description is given which applies to both missions, unless otherwise stated. The term "spacecraft" will refer to the ISS for Q-WEP and to the satellite for STE-QUEST.

\subsection{Measurement principle}

The Eötvös ratio $\eta(A, B)$ can be defined in terms of the differential acceleration between the two test bodies:

$$
\eta(A, B)=\frac{\left|a_{A}-a_{B}\right|}{g}
$$

where $g$ is the local value of the gravitational acceleration, $a_{A}$ and $a_{B}$ are the accelerations of test objects $A$ and $B$, respectively. In case of the AI, the two test bodies are ultra-cold ensembles of atoms. Thus, the instrument is a two species AI which measures the differential acceleration $\Delta a=a_{A}-a_{B}$ between the two atomic species. A direct readout of the differential acceleration is possible because the two species are prepared and interrogated simultaneously. The commonly used interferometer geometry for measuring accelerations in an AI is a Mach-Zehnder-like geometry. Here, the atomic wave packets are coherently split, redirected, and finally recombined via atom-light interactions. The light fields driving these interactions have an effective wave vector $k$. Between the atom-light interaction pulses the atoms propagate freely for a temporal duration of $T$. After recombining the atomic trajectories, the atom number at the interferometer output ports depends on the phase that the atoms acquire during the interferometer sequence. For an acceleration $a$ this phase shift scales as

$$
\phi_{a}=k a T^{2}
$$


where $k T$ denotes the scaling factor. The shot noise limit for an AI acceleration measurement scales as $1 / \sqrt{N}$ for the number of atoms $N$. Divided by the scaling factor this gives the sensitivity of the atom interferometer per shot. For $N=10^{6}$ and $k=(8 \pi / 780) \mathrm{nm}^{-1}$, the sensitivity of one interferometer per shot is $\sigma_{a}=$ $3 \cdot 10^{-11} T^{-2} \mathrm{~m} / \mathrm{s}^{2}$ leading to the differential sensitivity of $\sigma_{\Delta a}=\sigma_{a} \sqrt{2}$. The effective wave vector in this calculation corresponds to four photon recoils (see section 2.1.2). Integrating over a sufficiently large number of interferometer cycles leads to the targeted value of the Eötvös ratio.

The basic operation of the atom interferometer is determined by the choice of atom species. In both Q-WEP and STE-QUEST the ${ }^{85} \mathrm{Rb}^{87} \mathrm{Rb}$ pair is chosen as the only baseline which - due to its excellent common mode noise suppression - is expected to achieve the science requirement. This corresponds to a differential acceleration sensitivity of $\Delta a / a \sim 10^{-14}$ for Q-WEP, using a free evolution time $T \sim 1 \mathrm{~s}$ in the environment provided by the ISS, or $\triangle a / a \sim 10^{-15}$ for STE-QUEST, using a free evolution time $T \sim 3 \mathrm{~s}$ on a dedicated satellite (as stated later). This atom choice requires a rather complex cooling scheme, involving a dual trap, and an optical dipole trap (ODT). The ODT is necessary to allow for condensation of ${ }^{85} \mathrm{Rb}$ by Feshbach tuning, which otherwise is prevented by a negative scattering length.

The experimental apparatus will consist of a two dimensional (2D-) MOT loading the 3D-MOT on the chip inside a vacuum system, laser systems for cooling, coherent manipulating, and detecting the atomic ensembles of ${ }^{87} \mathrm{Rb}$ and ${ }^{85} \mathrm{Rb}$, the dipole laser for generating the crossed dipole trap, several coils to provide the magnetic fields and a magnetic shielding to suppress the effects of external stray fields.

The main steps of a typical measurement cycle are depicted in the following sections. The cycle time is expected to be $T_{C}=12 \div 18 \mathrm{~s}$, depending on the duration of the atom interferometry sequence, that will be longer in STE-QUEST.

\subsubsection{Preparation of the ultracold atomic ensembles}

The ultracold dual atomic sample is prepared within a ultra-high vacuum system. To load the desired large number of atoms, a combination of a $2 \mathrm{D}+\mathrm{MOT}$ and a $3 \mathrm{D}-\mathrm{MOT}$ is suggested. The 2D+MOT produces a slow, dual-isotope atomic beam towards the main physics chamber, using the continuous flow of atoms provided by an atomic dispenser. The trap consists of four coils and six laser beams carrying four wavelengths (cooling + repumping transitions for both isotopes).
The slow atoms from 2D-MOT beam are captured and cooled on a chip by the combination of magnetic and light fields forming a 3D-MOT in mirror configuration. Again the laser beams contain cooling and repumping frequencies for both isotopes; the chip produces the magnetic gradient for 3D trapping, and provides a reflecting surface for two of the four laser beams. Three pairs of Helmotz coils generate a uniform magnetic bias in the trapping region.

After loading $\sim 10^{9}$ atoms of both isotopes in the 3DMOT, the magnetic and optical configuration is changed into optical molasses for cooling below the Doppler limit; at the end of this phase the atoms are transferred to a purely magnetic trap. The latter pre-evaporates the ensembles and ensures a high transfer efficiency to the crossed dipole trap. After transferring the two species to the dipole trap, a strong magnetic field is necessary to drive ${ }^{85} \mathrm{Rb}$ through the Feshbach resonances. This finally allows for the condensation of the two species. After the release and some waiting time, the atomic ensembles are prepared in the magnetic insensitive sublevel via microwave preparation. After state preparation, the atoms are transferred in the interferometer position, which must be at a sufficient distance from the chip. Precise positioning can be accomplished by means of coherent momentum transfer via stimulated Raman transitions.

\subsubsection{Atom interferometry sequence}

After preparation of the dual ultra cold atomic sample, the interferometry sequence takes place. During this step, the prepared atomic ensembles are simultaneously subjected to three-pulse sequence, which forms the Mach-Zehnder-like interferometer geometry. Raman transitions between the two ground-state hyperfine levels, induced by counter-propagating photons, yield a net recoil of the atom, thus producing and entanglement between external and internal degrees of freedom of the atom. The internal state labelling allows for fluorescence detection of the two output ports of the interferometer. A double diffraction scheme (see Figure 2 ) is the baseline choice for the atom interferometry sequence, since it is insensitive to many effects due to its symmetric momentum transfer, has a superior magnetic immunity, is less sensitive to phase noise of the Raman laser, and allows for state-sensitive detection of all interferometer output ports [19].

The first and the third laser pulses induce Raman transitions with momentum transfer $\pm \hbar k$, with equal probability on opposite directions. The second pulse acts as a mirror by coupling each path to its opposite momentum state. Atoms on both $\pm \hbar k$ momentum states are in 


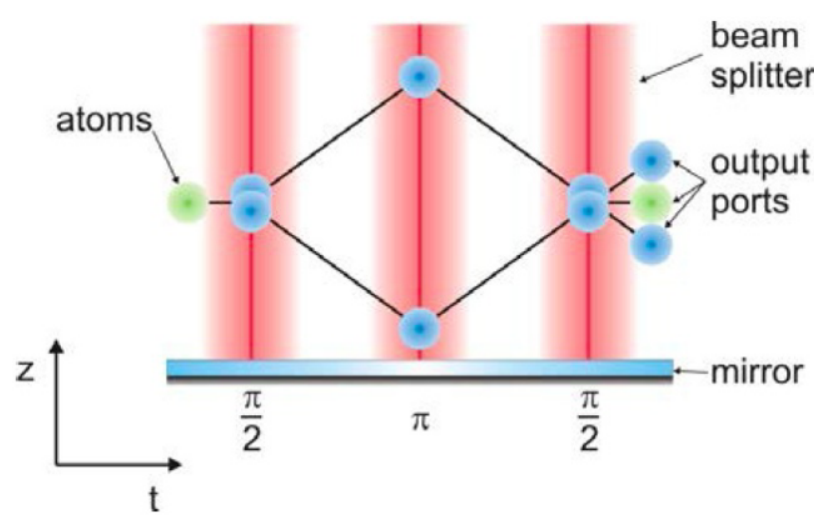

Figure 2: Illustration of the double diffraction scheme. The picture shows the implementation of a symmetric beam splitting in a MachZehnder like interferometer geometry. It depicts a free fall situation as provided by a space born environment.

the same internal state. After the first two beam splitter pulses, a blow away pulse removes atoms which were not properly excited.

\subsubsection{Detection}

The last step is the read out of the interferometer ports which corresponds to the measurement of the number of atoms in the two hyperfine levels of the $\mathrm{Rb}$ ground state. This is realized via fluorescence detection, employing a closed transition (typically the cooling transition) for both isotopes in order to produce strong fluorescence signals without losses of atoms. In one of the simplest implementations, the population of the upper level is measured first using the cooling transition (first output port) and collecting the fluorescence light on a photodiode. The measured atoms are optionally removed with a blow-away pulse. Then, a repumper transfers the atoms from the lower level to the upper level, and the population of the upper level is measured again using the cooling transition (second output port). This corresponds to a subsequent detection scheme.

The detection of both interferometer ports allows for a normalization which suppresses atom number fluctuations. However, frequency fluctuations of the probe laser on the time scale between the two detection pulses convert into noise in the normalized population measurement. Simultaneous detection of the interferometer output ports reduces the requirements on the detection laser line width and is then chosen as the baseline. After a waiting time in which the interferometer ports spatially separate, cooling and repumper light for the ${ }^{87} \mathrm{Rb}$ is simultaneously applied to the atoms. The scattered light is collected on photodetector (photodiode or CCD chip). In the next step the cooling and repumper tran-
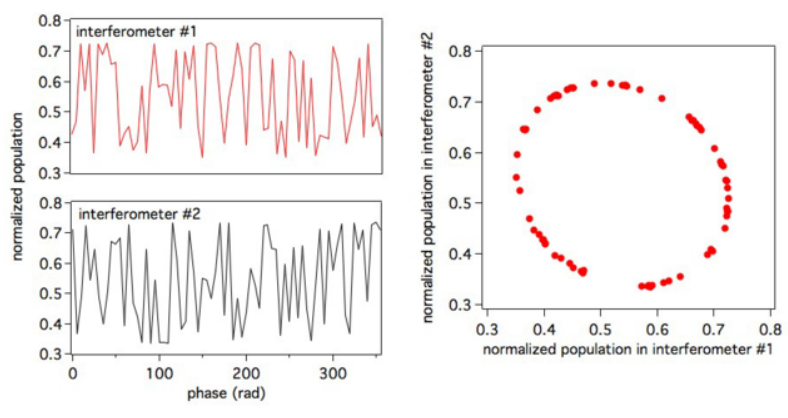

Figure 3: Left: fringes of a pair of simultaneous AIs where vibrationally induced RMS phase noise is larger than one period [21]. Right: Lissajous plot resulting from composition of the two fringes. The differential acceleration is determined from ellipse rotation angle.

sitions for ${ }^{85} \mathrm{Rb}$ are addressed simultaneously and the resulting fluorescence signal is read out via the same photodetector as for the ${ }^{87} \mathrm{Rb}$.

Since ${ }^{87} \mathrm{Rb}$ and ${ }^{87} \mathrm{Rb}$ have similar transition energies, there is a crosstalk when one of the isotopes is addressed. The ensembles with the two different isotopes spatially overlap and cannot be addressed independently. This will lead to a systematic error if it not accounted for. The detection yields four signals containing linear combinations of the four output port populations. As the scattering rates within the linear combinations are known, it is still possible to obtain the correct signal and thus eliminate the systematic error.

\subsubsection{Differential noise subtraction}

As the atom interferometers are sensitive to accelerations, the vibrational background has to be considered. Given the large scale factor of the atom interferometer to acceleration, vibrationally induced phase noise will span several interferometer fringe periods. A sensitive measurement of the relative phase of the two interferometers, that is proportional to the differential acceleration of the two atomic ensembles, can be obtained with the ellipse fitting method described in [20] to cancel common-mode phase-noise. The interference signal of one interferometer is plotted versus the interference signal of the other one (see Figure 3 ). The data then describe an ellipse and the relative phase shift can be obtained from its eccentricity and rotation angle. A high CMRR requires a precise matching of the scale factor of the two atom interferometers (see section 4.3)

\subsubsection{Mission duration}

For Q-WEP, assuming a single-shot sensitivity to differential acceleration in the range of $4.4 \cdot 10^{-11} \mathrm{~m} / \mathrm{s}^{2}$, and a cycle duration of $18 \mathrm{~s}$, an integration time of $10^{7} \mathrm{~s}$ 
corresponding to $5.6 \cdot 10^{5}$ experimental cycles will be required to reach the target $\sim 6 \cdot 10^{-14} \mathrm{~m} / \mathrm{s}^{2}$ accuracy on differential acceleration, i.e. $10^{-14}$ on the Eötvös parameter. Assuming a mission duty cycle of $40 \%$, the WEP test will require about ten months; including additional five months for the secondary objectives, and six months for commissioning and calibration, the whole mission duration is expected to be about 21 months.

For the STE-QUEST mission, a single-shot sensitivity to differential acceleration of the order of 3 . $10^{-12} \mathrm{~m} / \mathrm{s}^{2}$ is foreseen. Since the atom interferometry measurement is only performed at the perigee passage, where the gravitational acceleration is large enough, a few thousands orbits are required to reach the targeted sensitivity of $\sim 10^{-15} \mathrm{~m} / \mathrm{s}^{2}$. The mission duration is specified to 5 years.

\subsection{Improvements in space}

The potential single shot sensitivity of an AI in a Mach-Zehnder like configuration scales proportionally to the square of the free evolution time $T$. Thus, assuming a shot noise limited measurement, large $T$ is the key to build highly sensitive devices. In ground based experiments, the Earth's gravitational field accelerates the atomic ensembles requiring large vacuum systems to achieve free evolution times in the regime of seconds. In space, both the atoms as well as the reference platform and the retro-reflection mirror are in free fall, which in the ideal case leads to no displacement of the center of mass of the atomic trajectories with respect to the mirror. This enables free evolution times of several seconds within very compact vacuum setups as already demonstrated in ICE and QUANTUS and as also planned for STE-QUEST. In the latter, a free evolution time $T=5 \mathrm{~s}$ is foreseen. For Q-WEP, the target of testing the Eötvös ratio to one part in $10^{14}$ shall be reached with a free evolution time of $T=1 \mathrm{~s}$ and an integration of the signal over a few months. In principle these values are also possible within earth-bound $10 \mathrm{~m}$ towers. However, in this case the atomic ensembles cannot simply be released out of the trap as in microgravity but need to be launched. Due to the launch and earth's gravitational acceleration the center of mass of the atomic trajectories is displaced by about $10 \mathrm{~m}$ during the interferometer sequence with respect to the retro reflection mirror. The launching process needs to be precisely controlled, because even small differential displacement or velocities of the atomic ensembles will impose non negligible systematic errors. Additionally the apparatus needs to be carefully shielded against external disturbances e.g. magnetic stray fields over the whole size of $10 \mathrm{~m}$. In microgravity a launch is not necessary. Both the retro reflection mirror and the atomic ensembles after the release are in free fall with ideally no relative velocity, removing the need for the phase continuous and linear frequency chirp necessary in ground based AI gravimeters. Even more important, this also implies a compact setup since the vacuum chamber only needs to contain the splitting of the atomic trajectories in the order of several $\mathrm{cm}$ which reduces the volume in which external disturbances have to be suppressed. To reach the atomic temperatures necessary for a high signal to noise ratio and reduction of systematic errors an optical dipole trap is planned for Q-WEP and STE-QUEST. Microgravity eases the collocation of the two different atomic species in the same dipole trap. Relative to terrestrial matter-wave tests of the equivalence principle, Q-WEP and STE-QUEST also have the distinct advantage of the higher rotation frequency of the spacecraft relative to the Earth, which makes terms that go as $g\left(v^{2} / c^{2}\right)$ measurable.

Finally, Q-WEP can be also understood as a demonstrator for projects targeting a higher sensitivity onto the Eötvös ratio. The Q-WEP mission will provide a testbench for feasibility of the concept towards more challenging projects as STE-QUEST. This includes the evaluation of the source system, studies of the differential noise suppression and the introduction of free evolution times $T>1 \mathrm{~s}$.

\section{The atom Interferometer payload}

A block diagram of the atom interferometer is shown in Fig. 4. The instrument consists of three main blocks: control electronics, laser system and physics package. All systems are linked by optical interfaces (fibers) and electronic interfaces ( $r f$ lines, connectors).

\subsection{Electronic control system}

The electronics unit of the instrument provides an electronic interface to the spacecraft, and the entire instrument has a mechanical and thermal interface to the spacecraft. The electronics controls the complete measurement sequence. It switches and monitors the lasers, sets the frequency detuning of the lasers via AOMs and the currents through the various magnetic coils and the atom chip, and reads out the photodetector(s) which detects the measurement signal.

\subsection{Laser system}

The laser system is composed by two functionally identical sets of modules (for the two isotopes), including laser sources for cooling and trapping of atoms 


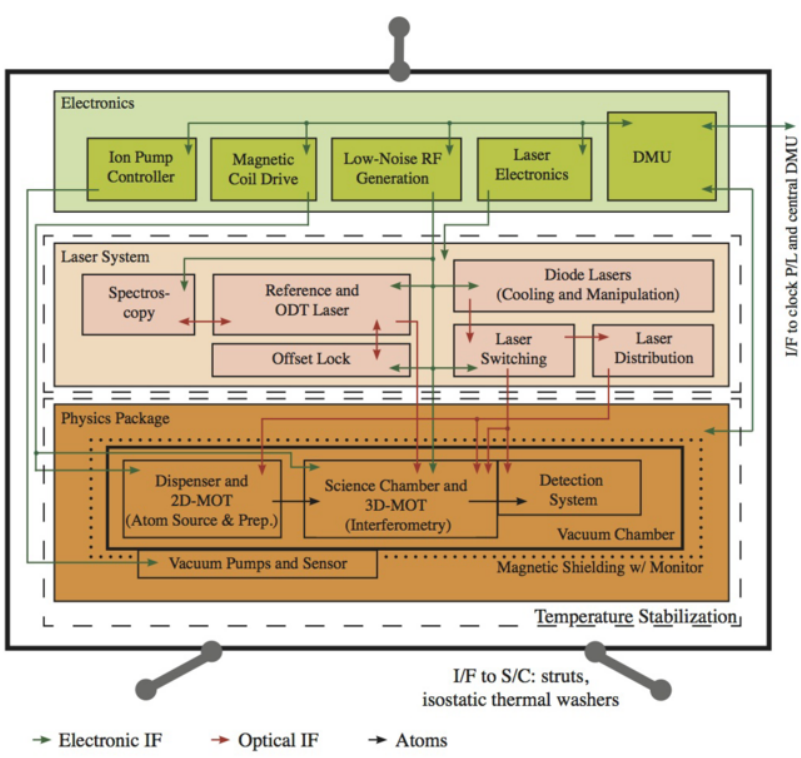

Figure 4: Block diagram of an atom interferometer for space use, and corresponding interfaces.

(MOT and ODT), state preparation and detection as well as manipulation of the atomic wave-packet in the interferometer. Laser radiation is delivered to the physics package through a distribution system including splitters, optical fibre couplers, modulators and shutters.

Most lasers require only small output powers $(<$ $200 \mathrm{~mW}$ ) at a wavelength close to the $\mathrm{Rb} D 2$ line at 780 $\mathrm{nm}$. For some of these lasers, frequency and power stability is a crucial requirement. Only the laser for the optical dipole trap operates at a different wavelength of $1.5 \mu \mathrm{m}$ and requires a considerable output power of $2 \mathrm{~W}$ total at the location of the atom (losses due to fiber coupling etc. have to be considered). Table 1 lists the main components of the laser package.

\subsection{Physics package}

The physics package includes an ultra-high vacuum system for the interferometric measurements with the two atomic species. This system comprises the coherent matter wave source, an atom chip combined with a dipole trap, the beam splitter unit comprising a highly stable, optically super flat retro reflector and the detection. The matter waves have to be generated and tracked in ultra-high vacuum and in a magnetically shielded environment with a precise and highly stable magnetic field. A dual-chamber design consisting of a 2D+MOT and a 3D-MOT/interferometer chamber, with differential pumping between the two regions, is considered for the vacuum system. The 2D-MOT provides short loading rate and large atom numbers in the 3D-MOT with a
Table 1: Basic sub-systems of the laser unit of the atom interferometer instrument.

\begin{tabular}{ll}
\hline Sub-system & Function / operation \\
\hline Reference & $\begin{array}{l}\text { Optical frequency reference for all } \\
\text { of other lasers }\end{array}$ \\
Near-resonant & $\begin{array}{l}\text { 2D/3D-MOT cooling, repumping, } \\
\text { blow-away, detection } \\
\text { lasers }\end{array}$ \\
Raman lasers & $\begin{array}{l}\text { atomic beam splitters } \\
\text { ODT laser }\end{array}$ \\
AOMs & $\begin{array}{l}\text { Frequency shifts of Rb lasers for various } \\
\text { functions (cooling, repump, pump, etc.) }\end{array}$ \\
Switches & $\begin{array}{l}\text { Distribution of beams to laser ports of } \\
\text { physics package in controlled timing }\end{array}$ \\
&
\end{tabular}

low background pressure in the main science chamber; these are necessary conditions to generate a BEC as required to achieve the main scientific objective of precise testing the WEP. Such design allows for the operation with a combination of ${ }^{87} \mathrm{Rb}$ and ${ }^{85} \mathrm{Rb}$, and is similar to the design in QUANTUS. Figure 5 shows a CAD drawing of the STE-QUEST physics package. Table 2 lists the elementary sub-systems of the physics package.

\subsection{Technology readiness}

The technology readiness of the single components is detailed in Table 3. Most components currently have TRL $3 \div 4$ but technology developments are ongoing or planned as detailed in the following paragraph.

Relevant laser technology is currently available in laboratory experiments, providing adequate support for atom cooling at adequate line widths with respect to output power to achieve the desired level of cooling.

The dual species atom interferometer using ${ }^{85} \mathrm{Rb}$ and ${ }^{87} \mathrm{Rb}$ has a TRL of 3 as no laboratory realization exists up to now. Nevertheless, ${ }^{87} \mathrm{Rb}$ BECs have been realized in drop tower experiments where a compact and robust setup was demonstrated (QUANTUS). Simultaneous laser cooling of ${ }^{87} \mathrm{Rb}$ and $\mathrm{K}$ atoms was demonstrated in zero-flight experiments (I.C.E.). Most of the components foreseen for STE-QUEST ATI are based on either QUANTUS/MAIUS or I.C.E. heritage with a current assigned TRL of $3 \div 4$. For these components, a TRL of $4 \div 5$ is expected to be reached until the end of 2013 as part of the corresponding ongoing projects. Most critical with respect to current TRL are the dual species reservoir (TRL $2 \div 3$ ), the atom chip (TRL 3), the magnetic shielding with high suppression factor (TRL 3), the Feshbach coil (TRL 3) and the science chamber (TRL $3 \div 4$ ). 
Table 2: Basic sub-systems of the physics package.

\begin{tabular}{|c|c|}
\hline Sub-system & Function / operation \\
\hline Atomic source & Generate $\mathrm{Rb}$ atomic vapor. \\
\hline 2D+MOT & $\begin{array}{l}\text { Slow atomic beam to load } \\
\text { 3D-MOT. }\end{array}$ \\
\hline Science chamber & $\begin{array}{l}\text { 3D-MOT, cooling to BEC, state } \\
\text { preparation, interferometer } \\
\text { sequence, detection. }\end{array}$ \\
\hline Atom chip & $\begin{array}{l}\text { Magnetic gradient for 3D-MOT, } \\
\text { rf-fields for evaporative cooling and } \\
\text { state transfer. }\end{array}$ \\
\hline Magnetic coils & $\begin{array}{l}\text { Bias fields for cooling, preparation, } \\
\text { and interferometer. }\end{array}$ \\
\hline $\begin{array}{l}\text { Rotating Raman } \\
\text { mirror }\end{array}$ & $\begin{array}{l}\text { Provides inertial reference for AI } \\
\text { acceleration measurement; tip-tilt } \\
\text { compensates for spacecraft rotations. }\end{array}$ \\
\hline Detection system & $\begin{array}{l}\text { Detect atomic fluorescence or } \\
\text { absorption signal. }\end{array}$ \\
\hline Vacuum pumps & $\begin{array}{l}\text { Provide adequate level of } \\
\text { background pressure. }\end{array}$ \\
\hline $\begin{array}{l}\text { Magnetic } \\
\text { shielding }\end{array}$ & $\begin{array}{l}\text { Attenuate external magnetic } \\
\text { disturbances of the interferometer. }\end{array}$ \\
\hline
\end{tabular}

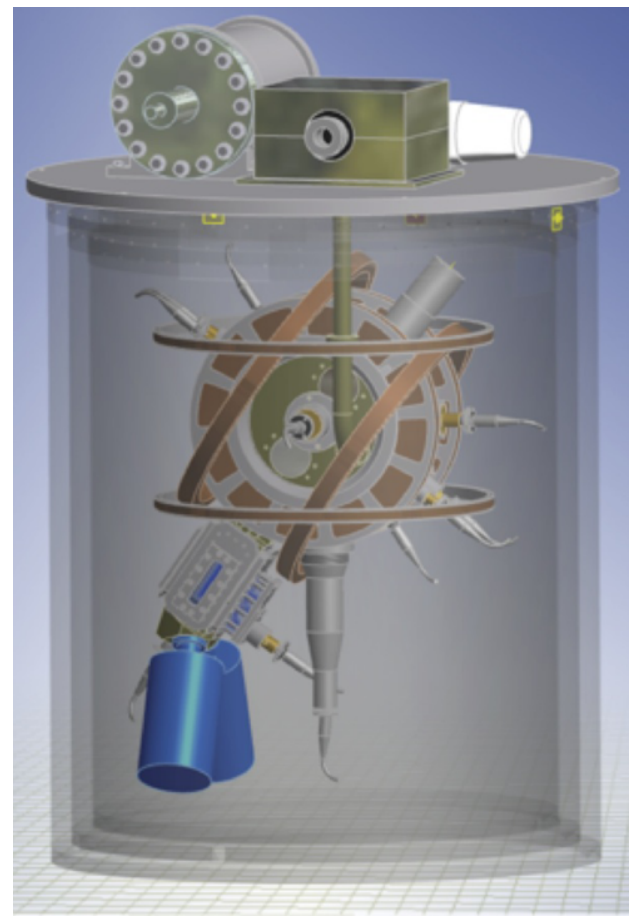

Table 3: Technology readiness level of the main subsystems.

\begin{tabular}{lll}
\hline Physics package & TRL & Heritage \\
\hline Science chamber & $3 \div 4$ & ACES \\
Atom chip & 3 & \\
2D-MOT & 4 & QUANTUS \\
Rb reservoir & $2 \div 3$ & ACES \\
Vaves & 4 & ACES \\
Telescopes & $4 \div 5$ & QUANTUS \\
Ion pump & 4 & QUANTUS \\
Getter pump & 4 & QUANTUS \\
UHV sensor & 4 & QUANTUS \\
Coils & 4 & QUANTUS \\
Thermal control & 4 & \\
CCD & 4 & \\
Photodiode \& optics & 4 & \\
Magnetic shield & 4 & ACES \\
\hline Laser system & TRL & Heritage \\
\hline Spectroscopy module & 4 & LASUS \\
780 nm diode lasers & $2 \div 3$ & FBH \\
Fiber switches & 4 & commercial \\
Fiber isolators & 4 & commercial \\
Fiber splitters & 4 & commercial \\
Optics & 4 & commercial \\
Shutters & 4 & commercial \\
Photodiodes & 4 & commercial \\
TEC & 4 & commercial \\
Offset stabilization module & $2 \div 3$ & LASUS \\
\hline & &
\end{tabular}
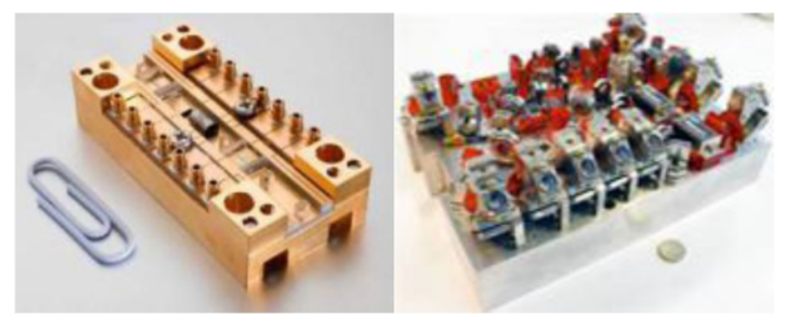

Figure 6: Left: micro-integrated master laser power amplifier (MOPA) module. Right: Rb switching and distribution module of the laser system for the QUANTUS-II experiment.

Figure 5: CAD drawing of the STE-QUEST physics package. 


\subsection{Ongoing, planned and proposed technology devel- opments}

Technology development is currently on-going as part of the I.C.E. and QUANTUS/MAIUS projects with expected TRL 4-5 at the end of 2013. In order to enhance the technology readiness, following activities are planned to be carried out in the upcoming STE-QUEST phases (after mission selection):

- Bread-boarding of the dual species ${ }^{85} \mathrm{Rb}^{8}{ }^{87} \mathrm{Rb}$ dispenser technology, including environmental testing according to STE-QUEST requirements

- Bread-boarding of a magnetic shielding demonstrating the suppression factor needed for STEQUEST

- Realization of a ${ }^{85} \mathrm{Rb}-{ }^{87} \mathrm{Rb}$ dual species atom interferometer on (enhanced) laboratory level taking into account foreseen technology for STE-QUEST, also a space-compatible design for the Feshbach coil cooling

- Demonstration activities (incl. environmental testing according to STE-QUEST requirements) with respect to the vacuum technology needed for realizing the vacuum chamber

- Further TRL development of all components with QUANTUS/MAIUS/I.C.E. heritage, especially performance of environmental tests taking into account STE-QUEST requirements.

These activities are expected to start in early 2014 and end in mid 2015, leading to TRL5 of all components. A demonstrator setup of a dual species ${ }^{85} \mathrm{Rb}-{ }^{87} \mathrm{Rb}$ ATI is currently being designed, and its realization is planned to start in 2014. This demonstrator will be realized on (enhanced) breadboard (EBB) level using mainly standard components. This setup is planned to fit into a drop-tower catapult capsule and performance tests in the drop tower facility will be carried out within 2014. An engineering model of the STE-QUEST ATI will be realized in the period from June 2015 to June 2018. The EM will be fully qualified and tested.

\section{Expected performance}

In the following, some of the most relevant sources of

\subsection{Quantum projection noise}

The shot noise limited sensitivity for a measurement of the differential acceleration $\Delta a$ between the two $\mathrm{Rb}$ isotopes is given by

$$
\sigma_{\Delta a}=\frac{1}{C} \frac{\sqrt{2}}{\sqrt{N} k T^{2}}
$$

per cycle or

$$
\sigma_{\triangle a, 1 s}=\frac{1}{C} \frac{\sqrt{2 T_{C}}}{\sqrt{N} k T^{2}}
$$

equivalent sensitivity at one second, where $N$ is the number of atoms, $T_{C}$ is the cycle duration, and $C$ is the interferometer contrast (see section 4.2). The factor $\sqrt{2}$ stems from the differential measurement between the two isotopes. The Eötvös ratio is then calculated by dividing $\Delta a$ by the local value of the gravitational acceleration $g$. Both the contrast and $g$ depend on the distance and orientation towards earth. In Q-WEP both parameters are nearly constant along the orbit, while in STE-QUEST they both vary significantly, which leads to a complex integration behavior. In particular, the attitude of the satellite will be kept inertial along the perigee passage. The contrast reduction will be discussed in section 4.2. For Q-WEP, with $T \simeq 1 \mathrm{~s}$, a contrast $\sim 1$ is foreseen, leading to a sensitivity per shot of $\sigma_{\Delta a} \simeq 4.4 \cdot 10^{-11} \frac{\mathrm{m}}{\mathrm{s}^{2}}$. In STE-QUEST, where the contrast with $T \simeq 3 \mathrm{~s}$ is expected to be reduced to $\sim 0.6$, the sensitivity per shot would be $\sigma_{\Delta a} \simeq 3 \cdot 10^{-12} \frac{\mathrm{m}}{\mathrm{s}^{2}}$

In the case of STE-QUEST, due to the large eccentricity of the orbit only few tens of interferometer cycles contribute to the integration on each orbit; afterwards the projection of local $g$ onto the sensitive axis becomes too small. An integrated sensitivity of $10^{-15}$ is reached after about 5 years.

\subsection{Interferometer contrast}

The signal of one interferometer is read out as the transition probability $P(\phi)=A_{0}+A_{s} \cos \phi$ for an offset $A_{0}$ and a signal amplitude $A_{s}$ depending on the phase shift $\phi$. In the latter the information about the acceleration $a$ is encoded by $\phi_{a}=k a T^{2}$. The contrast is defined as $C=A_{s} / A_{0}$ with ideally $C \sim 1$. Two effects have to be considered for estimating the interferometer contrast, the beam splitting efficiency and the averaging over spatially and velocity dependent phase shifts during detection. No reduction in $C$ is expected from beam splitting efficiency due to $T_{a t}=70 \mathrm{pK} \ll T_{\text {Doppler }} \sim 370 \mathrm{nK}$.

In the case of finite sizes of the atomic ensembles and finite temperatures each atom will experience a phase 


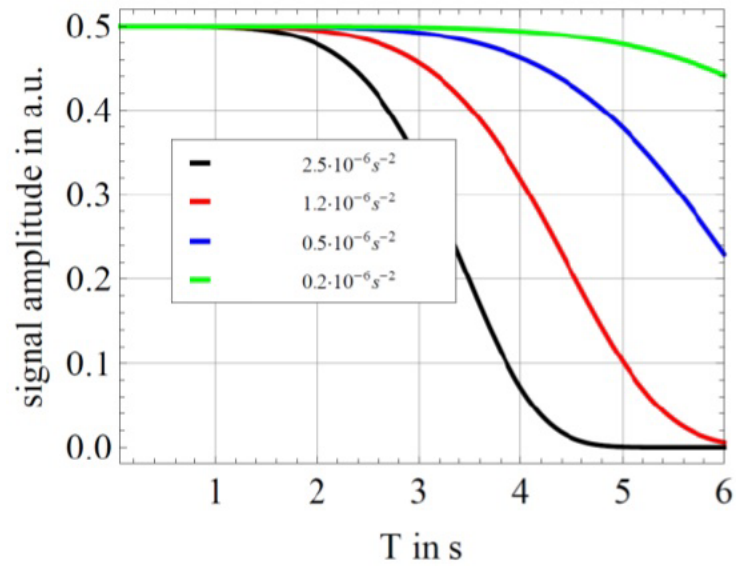

Figure 7: Contrast loss due to gravity gradient. The plot shows calculated signal amplitude versus interferometer time $T$ for different gravity gradients; atomic temperature is assumed to be $1 \mathrm{nK}$.

shift $\phi(r, v)$ depending on its velocity $v$ and location $r$. During detection the transition probability is averaged according to

$$
P_{t o t}=\int_{-\infty}^{+\infty} d r d v f(r) g(v) P_{S}(\phi(r, v))
$$

Contrast reduction may be induced by either rotations or gravity gradients. The amplitude reduction due to rotations $\Omega_{x}$ and $\Omega_{y}$ couples in via the Sagnac effect, see section 4.5; as long as reference frame angular velocity is kept at the $10^{-6} \mathrm{rad} / \mathrm{s}$ level (mirror rotation in Q-WEP; inertial attitude of the STE-QUEST satellite), $C>96 \%$, i.e. the effect of rotations on contrast is negligible. Figure 7 shows the interferometer contrast versus the free evolution time $T$, for different values of the gravity gradient $T_{z z}$ along the axis, assuming a temperature of about $1 \mathrm{nK}$. At the closest perigee passage of STE-QUEST the contrast is $C \simeq 0.6$ with $T=3 \mathrm{~s}$. The gradients $T_{x x}$ and $T_{y y}$ couple into the interferometer only via spurious rotations. Combined with rotations on the $10^{-6} \mathrm{rad} / \mathrm{s}$ the impact onto the contrast is negligible. This effect would matter for free evolution times $T$ above several tens of seconds. Cross coupling of the gradients $T_{z x}, T_{z y}$ into the sensitive axis of the interferometer with respect to the contrast can also be considered as negligible.

\subsection{Scale factor calibration}

The WEP test relies on a precise tuning of the scale factor $S$ for the two atom interferometry accelerometers, i.e. $S_{85}=k_{85} T_{85}^{2}=S_{87}=k_{87} T_{87}^{2}$. Any mismatch $\Delta S=S_{85}-S_{87}$ in the scale factors will induce an additional phase term $\Delta \phi=a \Delta S$. If the residual acceleration $a$ is limited to $\sim 4 \cdot 10^{-7} \mathrm{~m} / \mathrm{s}^{2}$, the scale factors must be equalized with $\sim 10^{-9}$ precision. This means measuring the relative optical frequency of Raman lasers for ${ }^{85} \mathrm{Rb}$ and ${ }^{87} \mathrm{Rb}$ to $\sim 200 \mathrm{kHz}$, and the time interval between Raman pulses with ns accuracy.

In principle, with the ${ }^{85} \mathrm{Rb}-{ }^{87} \mathrm{Rb}$ pair the effective wave vectors can be made equal for the two interferometers. This would allow a matching of the scale factors using the same timing i.e. by passing all Raman beams in the same AOM for pulse shaping which would automatically meet the requirements on timing accuracy.

In fact, for counter-propagating Raman beams with frequencies $v_{1}$ and $v_{2}$

$$
k=\frac{2 \pi}{c}\left(v_{1}+v_{2}\right)=\frac{2 \pi}{c}\left[2\left(v^{0}+\delta\right)+\Delta\right]
$$

where $\Delta$ is the hyperfine splitting and $\delta$ is the detuning of Raman laser 1 from the optical resonance $v^{0}$ of the $D 2$ line. Requiring the wave vectors to be equal for the two interferometers, i.e. $k_{87}=k_{85}$, implies that

$$
\delta_{85}=\delta_{87}+\frac{\Delta_{87}-\Delta_{85}}{2}+v_{87}^{0}-v_{85}^{0}
$$

\subsection{Acceleration noise}

In the case of stationary, zero-mean accelerations as random vibrations, the noise sources are properly described by a power spectral density (PSD) $S(f)$. The resulting rms differential acceleration noise of the dual atom interferometer is calculated as $\sigma_{\Delta a}=$ $\int_{0}^{\infty} d f\left|H_{\Delta a(f)}\right|^{2} S(f)$. Here, $H_{\Delta a}(f)$ describes the transfer function for acceleration noise in the dual atom interferometer [22]. With deterministic signals instead as periodic signals, for which the PSD is not well defined, one can calculate an rms value over frequency intervals. The rms noise can by multiplied by the transfer function $H_{\Delta a}(f)$ to give the effect $\sigma_{\Delta a}$ onto differential acceleration. The transfer function from acceleration noise to differential acceleration noise is calculated as $H_{\Delta a}(f)=\left|H_{a 87}(f)-H_{a 85}(f)\right| /\left(k T^{2}\right)$. Each $H_{a i}(f)$ with $i=85,87$ represents the single species transfer function from acceleration noise to an interferometer phase:

$$
\begin{gathered}
H_{a i}(f)=-\frac{4 \Omega_{R i} k_{i}}{\Omega_{R i}^{2}-(2 \pi f)^{2}} \frac{\sin \pi f T_{i}}{(2 \pi f)^{2}} . \\
\cdot\left\{\sin \left[\pi f\left(T_{i}-2 \tau_{i}\right)\right]+\frac{2 \pi f}{\Omega_{R i}} \cos \left(\pi f T_{i}\right)\right\}
\end{gathered}
$$

As one can see, the transfer function does not only depend on the effective wave vector $k_{i}$, but also on the Rabi frequency $\Omega_{R i}$, the duration of a beam splitter $\pi$ pulse $\tau_{i} \approx \pi /\left(2 \Omega_{R i}\right)$, and the free evolution time $T$. 


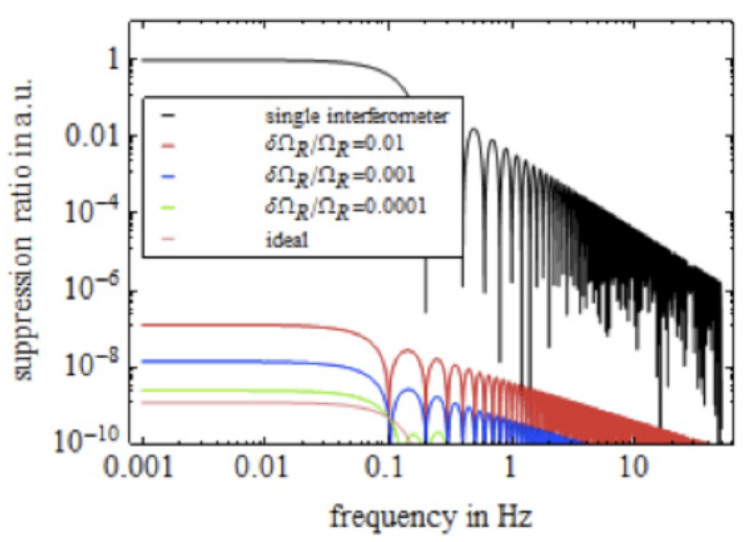

Figure 8: Suppression ratio for linear accelerations dependent on the Rabi frequency mismatch $\Delta \Omega_{R} / \Omega_{R}$. The effective wave vectors for all plots are matched to 1 part in $10^{9}$.

Pulse durations and free evolution times will be controlled by the same AOM for both species leading to $T_{87}=T_{85}=T$ and $\tau_{87}=\tau_{85}$ which is assumed to be $\sim 100 \mu \mathrm{s}$. The Rabi frequency of $\sim 15.7 \mathrm{kHz}$ needs to be adjusted to match the transfer functions and thus maximize the suppression ratio in the differential signal. Matching the effective wave vectors by $\Delta k / k \sim 10^{-9}$ leads to the plot in Figure 8. For a Rabi frequency mismatch of $\Delta \Omega_{R} / \Omega_{R} \sim 10^{-\alpha}$ with $\alpha=2,3,4$ the asymptotic behavior for $f<0.1 \mathrm{~Hz}$ is $H_{\Delta a}(f) \sim 2.5 \cdot 10^{-(5+\alpha)}$ and the envelope for $f>0.1 \mathrm{~Hz}$ is $\sim 8 \cdot 10^{(7+\alpha)} \mathrm{Hz} / f$.

The requirements on acceleration noise depend on the actual values of the effective wave vector and Rabi frequency mismatch. Under our assumptions, $k$ compensation to $10^{-9}$ and Rabi frequency matching to $10^{-4}$ would lead to a suppression ratio of $2.5 \cdot 10^{-9}$.

Stochastic, zero-mean accelerations or vibrations affect the sensitivity and the resulting differential acceleration $\sigma_{\Delta a}$ shall be kept below the single shot sensitivity of $\sim 10^{-12} \mathrm{~m} / \mathrm{s}^{2}$. This is compatible with the requirement to have the PSD of acceleration noise lower than $0.5 \cdot 10^{-3} \mathrm{~m} /\left(\mathrm{s}^{2} \mathrm{~Hz}^{1 / 2}\right)$ or to have the rms of periodic accelerations lower than $0.5 \cdot 10^{-3} \mathrm{~m} / \mathrm{s}^{2}$.

However, since the measurement is taken with a fixed repetition rate $1 / T_{\text {rep }}$, the aliasing effect will downconvert noise at the multiples of the repetition rate to low frequencies. For long averaging times $N \cdot T_{\text {rep }}$ the Allan variance is more appropriate than the one-sample average. The Allan variance of differential acceleration measurement will be given by

$$
\sigma_{\Delta a}^{2}\left(N T_{r e p}\right)=\frac{4}{N^{2}}
$$

$$
\cdot \int_{0}^{\infty} \frac{\sin ^{4} \pi f N T_{r e p}}{\sin ^{2} \pi f T_{r e p}}\left|H_{\Delta a}(f)^{2}\right| S_{a}(f) d f
$$

In order to derive requirements on vibration noise, the Allan variance can be evaluated for different types of acceleration noise spectra (white acceleration noise, Flicker acceleration noise etc.). The target Q-WEP differential acceleration sensitivity requires the PSD of acceleration noise to be smaller than

$$
\begin{aligned}
\frac{5 \cdot 10^{-5}}{\sqrt{f}} \frac{m}{s^{2}} & \text { for } 1 \mathrm{mHz}<f<10 \mathrm{mHz} \\
5 \cdot 10^{-4} \frac{m}{s^{2} \sqrt{H z}} & \text { for } 0.02 \mathrm{~Hz}<f<2 \mathrm{~Hz} \\
3.5 \cdot 10^{-4} \sqrt{f} \frac{m}{s^{2} \mathrm{~Hz}} & \text { for } 2 \mathrm{~Hz}<f<100 \mathrm{~Hz}
\end{aligned}
$$

Figure 9 shows the calculated contribution of vibrations to the Allan variance of the Q-WEP differential acceleration measurement, assuming either the acceleration noise spectrum of eq. 10 or a typical noise spectrum on the ISS.

An additional limit is set by the requirement to keep the Doppler shift much smaller than the Rabi frequency. This means $k \cdot \delta a_{r m s} /(2 \pi f)<\Omega_{R} \sim 1.6 \mathrm{kHz}$ and translates to having the PSD of acceleration noise below $10^{-3} \mathrm{f} /(1 \mathrm{~Hz}) \mathrm{m} /\left(\mathrm{s}^{2} \mathrm{~Hz}^{1 / 2}\right)$ and to have the rms of periodic accelerations below $10^{-3} \mathrm{f} /(1 \mathrm{~Hz}) \mathrm{m} / \mathrm{s}^{2}$.

The requirements on vibration noise might be relaxed by one order of magnitude if a 10-fold higher Rabi frequency is employed, i.e. by reducing the duration of Raman pulses, since the effect of both Doppler shift and Rabi frequency on scale factor depend on the ratio $\tau / T$.

Periodic, low frequency or DC accelerations like effects at the harmonics of the orbital period (drag, gravity gradient etc.) can cause systematic errors. The resulting differential acceleration shall be kept below the target accuracy level, i.e. $10^{-14} \mathrm{~m} / \mathrm{s}^{2}$ for Q-WEP and $10^{-15} \mathrm{~m} / \mathrm{s}^{2}$ for STE-QUEST. In STE-QEST, assuming a CMRR of $2.5 \cdot 10^{-9}$, this converts into the requirement to have the rms of periodic accelerations below $4 \cdot 10^{-7} \mathrm{~m} / \mathrm{s}^{2}$. An upper limit to the systematic error arising from scale factor mismatch can be set by measuring the residual spurious low frequency axial accelerations. In principle, the atom interferometer itself could be employed to measure the residual acceleration along the sensitive axis. However, this is only possible provided that the fringe visibility is recovered, either by isolating the retro-reflecting Raman mirror from vibrations, or by measuring the vibration noise with a mechanical accelerometer in the range $0.1 \div 100 \mathrm{~Hz}$. The free-fall time $T$ can should be reduced in order to limit the overall phase noise to $\sim 10$ cycles, if the mechanical ac- 


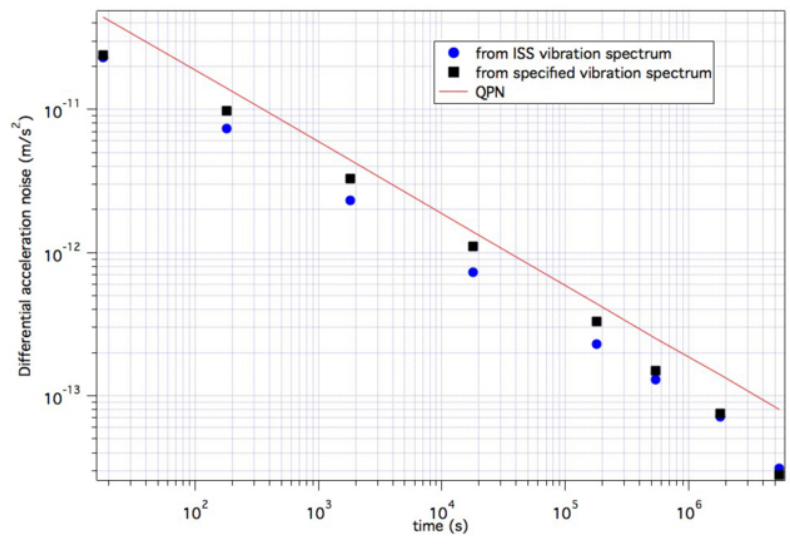

Figure 9: Calculated Allan variance of differential acceleration for the QPN limit (red line), for vibration noise of the spectrum in eqn. 10, and for typical vibration noise on the ISS. The cycle time was taken to be $18 \mathrm{~s}$.

celerometer is employed, or otherwise to about one cycle. Such measurement should be done during the calibration stage, and possibly repeated periodically during the science runs by interleaving linear acceleration measurement with WEP test measurements. Since the order of magnitude of residual low frequency accelerations will be around $10^{-5} \mathrm{~m} / \mathrm{s}^{2}$, a precision of $10^{-7} \mathrm{~m} / \mathrm{s}^{2}$ or better will be easily obtained, mostly limited by the stability of the spurious acceleration itself.

In principle, the precise knowledge of the axial acceleration would give access to a simple method to reduce the systematic error from scale factor mismatch, instead of a simple estimation of an upper limit. In fact, it is possible to apply an effective axial acceleration to the reference frame by chirping the frequency of Raman lasers during the atom interferometry sequence, just like in terrestrial gravimeters. Mitigation of biases from scale factor mismatch would consist in chirping the Raman lasers in order to reduce the net acceleration below $10^{-7} \mathrm{~m} / \mathrm{s}^{2}$.

\subsection{Error terms from gravity gradients and rotations}

The atom interferometry phase shift induced by gravity gradients and rotations can be calculated following the approach of [23]. Most of the phase shifts are cancelled in the differential interferometry since the wave vector $k$ and the free evolution time $T$ are the same for both isotopes. Moreover, the symmetric splitting of the double diffraction eliminates the phase shifts $\propto h k^{2} / m$ within a single interferometer ( $h$ : Plancks constant, $m$ : atomic mass). Remaining errors arise due to differential velocities and positions between the atomic ensembles. Relative displacement of the atomic ensembles along the sensitive axis couples with the gravity gradient: assuming just the effect of the Earth gravity gradient $\sim 2.5 \cdot 10^{-6} \mathrm{~s}^{-2}$, in order to limit the differential acceleration to $10^{-15} \mathrm{~m} / \mathrm{s}^{2}$ the average differential displacement must be kept below $1 \mathrm{~nm}$. Similarly, the Coriolis acceleration couples angular velocities with the difference of bulk velocity of the atomic clouds in the direction transverse to the sensitive axis. Knowledge of the differential transverse velocity at the $\mathrm{nm} / \mathrm{s}$ level is required for differential acceleration accuracy at the $10^{-15}$ level, provided that the angular rate is kept within $\sim 10^{-6} \mathrm{rad} / \mathrm{s}$. The angular rates will be limited via attitude control in STE-QUEST. For Q-WEP a counterrotation of the retro-reflecting Raman mirror will be required in order to compensate for the $\sim 1 \mathrm{mrad} / \mathrm{s}$ rotation rate of the ISS [24].

\subsection{Magnetic fields}

The operation of the AI requires a small, uniform bias magnetic field for the separation of the Zeeman $m_{F}=0$ sublevel. In the presence of a gradient $\nabla B$ in the magnetic field, an additional acceleration signal results, which is proportional to the bias field, to the gradient, as well as to the coefficient $\alpha$ of second-order Zeeman shift. With a bias field $B_{0}=100 \mathrm{nT}=1 \mathrm{mG}$, the differential acceleration signal is $\Delta a=7 \cdot 10^{-5} \nabla B$, where $\Delta a$ is in $\mathrm{m} / \mathrm{s}^{2}$ and $\nabla B$ is in $\mathrm{T} / \mathrm{m}$. In order to keep the effect below $10^{-15} \mathrm{~m} / \mathrm{s}^{2}$, the gradient should be kept below $\sim 0.1 \mathrm{nT} / \mathrm{m}$, which is technically challenging; such requirement can be released if the effect is cancelled to some degree by alternating the measurement between the different hyperfine levels.

Fluctuations of the magnetic fields will couple into the interferometer signal if a gradient is present. If this gradient is inherently smaller than the stated $0.1 \mathrm{nT} / \mathrm{m}$ fluctuations of the $0.1 \mu \mathrm{T}$ interferometry offset field on a percent level would be negligible. By alternating the hyperfine states the atomic ensembles are in before entering the beam splitter pulse sequence the impact of a remaining gradient can be suppressed. Assuming a 1\% change of the interferometry offset fields over the baseline of $12 \mathrm{~cm}$ the gradient would be $8.3 \mathrm{nT} / \mathrm{m}$. Fluctuations on a $10 \%$ level from cycle to cycle would result in an acceleration noise of $1.1 \cdot 10^{-13} \mathrm{~m} / \mathrm{s}^{2}$ below the shot noise limit by a factor of $\sim 10$ and thus are negligible.

Additional constraints to the magnetic field gradients have to be considered after the release from the ODT. At this point the Feshbach field with $B_{0} \sim 155 \mathrm{G}$ is still switched on and the atoms are still in the magnetic sensitive sub states $\left|F=2, m_{f}=2\right\rangle$ for ${ }^{87} \mathrm{Rb}$ and $\left|F=3, m_{f}=3\right\rangle$ for ${ }^{85} \mathrm{Rb}$ for a time $t \sim 0.01 \mathrm{~s}$. 
Any gradient combined with the linear and second order Zeeman effect will induce an acceleration. Until the ensembles are transferred to the magnetic insensitive sub state a magnetic field gradient $\delta B$ combined with the linear Zeeman effect will lead to a differential acceleration producing a differential velocity of $\Delta v \simeq-150 \mu \mathrm{m} / \mathrm{s} \cdot \mathrm{m} / \mathrm{G} \cdot \delta B$ and a differential displacement of $\Delta r \simeq-75 \mu \mathrm{m} \cdot \mathrm{m} / \mathrm{G} \cdot \delta B$. The differential acceleration due to the quadratic Zeeman effectleads to a differential velocity of $\Delta v=-533 \mu \mathrm{m} / \mathrm{s} \cdot \mathrm{m} / \mathrm{G} \cdot \delta B$ and a differential displacement of $\Delta r=-267 \mu \mathrm{m} \cdot \mathrm{m} / \mathrm{G} \cdot \delta B$. Propagation in the offset field of $1 \mathrm{mG}$ with a duration of $1 \mathrm{~s}$ in the magnetic insensitive states leads to negligible contributions of $\Delta v_{0}=0.1 \mathrm{pm} / \mathrm{s}$ and $\Delta x_{0}=0.01 \mathrm{pm}$ for a gradient of $10^{-4} \mathrm{G} / \mathrm{m}$.

\subsection{Wave front distortions}

The wave fronts of the beam splitting light fields lead to statistical and systematic errors, and also affect the interferometer's contrast. The important part is the retro reflection since one of the two beam splitter light fields is retro reflected while the other is not. The expansion of the atomic ensemble combined with the inhomogeneity of the effective wave front leads to systematic phase shift. To quantify this effect, a finite curvature of the effective wave front will be considered.

Two effects have to be discussed, the initial collimation of the Raman beams and the disturbances imprinted in the retro reflection path. For the retro reflection a defocus imprinted by the optics onto the laser beam is assumed. If this defocus leads to an effective wave front curvature $R$ a phase shift

$$
\phi_{r r}=k \frac{k_{B} T_{a t}}{R m} T^{2}
$$

appears for a single interferometer [25]. The acceleration error $a_{r r}=\phi_{r r} /\left(k T^{2}\right)$ is independent on $T$, but is generally different for the two AIs. This systematic effect imposes stringent requirements on both the atomic temperatures and on the quality of Raman beams wave front. With $R=250 \mathrm{~km}$ and $T_{a t}=1 \mathrm{nK}$ the differential acceleration error is $\Delta a_{r r} \simeq 6 \cdot 10^{-16} \mathrm{~m} / \mathrm{s}^{2}$.

An imperfect initial collimation on the beam splitter light field leads to the same effect. This bias could possibly be calibrated by tuning the effective temperature since it is expected to be stable in time.

\subsection{Mean field}

The atomic interactions will induce a phase uncertainty depending on the beam splitting quality of the first $\pi / 2$-pulse and the density of the atomic ensembles
[26]. The differential phase between the two trajectories with a shot noise limited atom number difference in the two trajectories is thus

$$
\omega_{m f}(t)=2 \pi \frac{\mu V(0)}{h \sqrt{N} V(t)}
$$

Here, $V(t)$ is the volume of the atomic ensemble, $N$ is the atom number, $h$ is Plancks constant, $\mu=n(0) U$ is the chemical potential with the initial peak atom density $n(0)$ and the interaction parameter $U=\frac{h a_{s c}}{\pi m}$ for the scattering length $a$ and the atomic mass $m$. The initial peak density is defined as $n(0)=N\left(\frac{m \omega^{2}}{2 \pi k_{B} T_{a t}}\right)^{3 / 2}$ with the isotropic trap frequency $\omega$, the Boltzmann constant $k_{B}$ and the atomic temperature $T_{a t}$. The parameters are assumed to be $\omega=1 \mathrm{~Hz}, V(0)=4 \pi(1.5 \mu \mathrm{m})^{3} / 3, T_{a t}=$ $0.07 \mathrm{nK}$ with the scattering lengths $a_{s c, 87}=100 a_{0}$ for ${ }^{87} \mathrm{Rb}, a_{s c, 85}=-450 a_{0}$ for ${ }^{85} \mathrm{Rb}, a_{s c, 85 / 87}=213 a_{0}$ for $85 \mathrm{Rb}-{ }^{87} \mathrm{Rb}$ interactions and the Bohr radius $a_{0}$. Since the atomic ensembles are kicked away from atom chip, the delay between opening the optical dipole trap and starting the beam splitter pulse sequence is $1 \mathrm{~s}$. For a free evolution time $T=5 \mathrm{~s}$ and integration over the frequency shift the acceleration errors are $\delta a_{87}=$ $8.3 \cdot 10^{-18} \mathrm{~m} / \mathrm{s}^{2}$ for ${ }^{87} \mathrm{Rb}, \delta a_{85}=-3.6 \cdot 10^{-18} \mathrm{~m} / \mathrm{s}^{2}$ for ${ }^{85} \mathrm{Rb}$, and $\delta a_{87-85}=1.7 \cdot 10^{-17} \mathrm{~m} / \mathrm{s}^{2}$ for the interactions between ${ }^{87} \mathrm{Rb}$ and ${ }^{85} \mathrm{Rb}$. This sums up to $\delta a=$ $-1 \cdot 10^{-17} \mathrm{~ms}^{2}$. Beam splitting accuracy was assumed to be $1 \sqrt{N}=0.001$ for an atom number $N=10^{6}$. The trap frequency considered in this calculation is an effective trap frequency derived from the expansion behaviour after the delta kick and extrapolation versus zero.

\subsection{Detection efficiency unbalance}

The phase difference between the two atom interferometers is retrieved as the phase angle $\Delta \phi$ of a Lissajous ellipse, using a Bayesian estimator. The target $10^{-15} \mathrm{~m} / \mathrm{s}^{2}$ corresponds to measuring the ellipse phase angle with an accuracy of $1 \mu \mathrm{rad}$. One possible source of bias in the determination of the ellipse phase angle is from detection unbalance between the two output channels of the atom interferometer. The coordinates $\{x ; y\}$ of a point in the ellipse are determined from the normalized number of atoms in one of the two input states for the two atom interferometers respectively. The population $n_{i j}$ of the $i$-th state $(i=1,2)$ is proportional to the amplitude $S_{i j}$ of the corresponding detection signal, i.e. $n_{i j}=\eta_{i j} S_{i j}$ where $j=1,2$ for ${ }^{87} \mathrm{Rb}$ and ${ }^{85} \mathrm{Rb}$, respectively. If the detection efficiencies of the two channels are not equal, i.e. $\eta_{j}=\eta_{1 j} / \eta_{2 j} \neq 1$, then the Lissajous plot represents a distorted ellipse. The phase angle obtained from Bayesian fit to a regular ellipse will depend 
on the $\eta_{j}$ values. Assuming for simplicity $\eta_{i}=\eta_{j} \equiv \eta$, the $\Delta \phi(\eta)$ function shows a parabolic extreme around $\eta=1$. The curvature is such that the target $1 \mu \mathrm{rad}$ accuracy on $\Delta \phi$ requires to determine $\eta$ at the level of $0.3 \%$. While the absolute calibration of detection efficiencies at this level might be not obvious, it is possible to include the unknown $\eta_{j}$ values into the model for the Bayesian estimator.

\subsection{Detection laser linewidth}

Frequency jitter of the detection light changes the scattering rate. During the detection sequence the scattering rate has to be constant to allow for normalization if a subsequent read out scheme for the interferometer ports is chosen. Assuming typical values for detuning and intensity for the probe laser, a duration of the detection sequence of $10 \mathrm{~ms}$ and pulse durations of $800 \mu \mathrm{s}$, a frequency error of less than $2 \mathrm{kHz}$ is required to stay below shot noise by a factor of three. Further assuming white frequency noise, this value corresponds to a spectral noise density of $S_{v}=3556 \mathrm{~Hz}^{2} / \mathrm{Hz}$. The full width half maximum of the Lorentzian amounts to $11.2 \mathrm{kHz}$. This condition is relaxed if both interferometer ports are detected simultaneously. In this case, the requirement on the linewidth can be relaxed to $100 \mathrm{kHz}$.

\subsection{Summary of sensitivity and accuracy limits}

The most relevant sources of systematic and statistical errors are lister in tables 4 and 5, respectively. Both for Q-WEP and STE-QUEST, calculated statistical errors are in line with the initial assumption of a shot noise limited sensitivity. For STE-QUEST, systematic errors sum up to $1.7 \cdot 10^{-14} \mathrm{~m} / \mathrm{s}^{2}$ which has to be weighted by the gravitational acceleration. According to the orbit and integration simulations the minimum value of the gravitational acceleration onto the sensitive axis is expected to be $3 \mathrm{~m} / \mathrm{s}^{2}$ leading to an accuracy in the Eötvös ratio of $\sim 6 \cdot 10^{-15}$. Error terms that are not related to the Earths gravitational field as those related Raman laser front can be calibrated for during the time around apogee. This corresponds to roughly half the estimated contribution to the systematic error budget and would result in an accuracy of $\sim 3 \cdot 10^{-15}$. The other terms related to inertial motion will be assessed by auxiliary data from orbit determination and the spacecraft. In QWEP, estimated error biases sum up to $7.3 \cdot 10^{-14} \mathrm{~m} / \mathrm{s}^{2}$.

\section{Conclusions}

Atom interferometry in space will open new frontiers for precision measurements in fundamental physics and
Table 4: Summary of most relevant systematic errors; conditions and numbers in square brackets refer to STE-QUEST, otherwise to QWEP.

\begin{tabular}{lll}
\hline Noise source & Conditions & $\delta a\left(\mathbf{1 0}^{-15} \mathbf{~ m} / \mathbf{s}^{2}\right)$ \\
\hline Gravity gradient & $\Delta z=4[1.1] \mathrm{nm}$ & $10[2.6]$ \\
& $\Delta v_{z}=1.5[0.3] \mathrm{nm} / \mathrm{s}$ & $4[3.5]$ \\
Coriolis accel. & $\Delta v_{x, y}=1.5[0.3] \mathrm{nm} / \mathrm{s}$ & $3[0.6]$ \\
Other inertial & $\Delta x=2 \mu \mathrm{m}[1 \mathrm{~nm}]$ & $6[0.06]$ \\
terms & $\Delta y=0.8 \mu \mathrm{m}[1 \mathrm{~nm}]$ & $0.9[0.0016]$ \\
Magnetic fields & $\nabla B<0.3[0.1] \mathrm{nT} / \mathrm{m}$ & $10[1]$ \\
Mean field & $t_{0}=1 \mathrm{~s}, \mathrm{see} 4.8$ & $15[0.01]$ \\
Raman laser & $T_{a t}=1[0.07] \mathrm{nK}$ & \\
wavefront & $R=900[250] \mathrm{km}$ & $4[0.6]$ \\
Spurious accel. & $\frac{\delta k}{k}=10^{-9}$ & \\
& $\frac{\delta \Omega_{R}}{\Omega_{R}}=10^{-3}\left[10^{-4}\right]$ & $7.5[1]$ \\
& $a=6[4] \cdot 10^{-7} \mathrm{~m} / \mathrm{s}^{2}$ & \\
Detection & $|\eta-1|<0.003$ & $<10[1]$ \\
efficiency & & \\
\hline
\end{tabular}

Table 5: Summary of most relevant statistical errors; the third column represent the differential acceleration noise per interferometer cycle; conditions and numbers in square brackets refer to STE-QUEST, otherwise to Q-WEP.

\begin{tabular}{|c|c|c|}
\hline Noise source & Conditions & $\delta a\left(\mathbf{1 0}^{-12} \mathbf{~ m} / \mathbf{s}^{2}\right)$ \\
\hline QPN & $10^{6}$ atoms & \\
\hline & $C=1[0.6]$ & 44 [2.9] \\
\hline Vibrations & $\begin{array}{l}\frac{\delta k}{k}=10^{-9} \\
\frac{\delta \Omega_{R}}{\Omega_{R}}=10^{-3}\left[10^{-4}\right] \\
S_{a}(f) \text { as in eq. } 10\end{array}$ & $24[2.4]$ \\
\hline Magnetic fields & $\begin{array}{l}B_{0}=100 \mathrm{nT} \\
\nabla B=40 \mathrm{nT} / \mathrm{m} \\
10 \% \text { fluct. } / \text { cycle }\end{array}$ & $0.5[0.1]$ \\
\hline $\begin{array}{l}\text { fluct. differential } \\
\text { position/velocity }\end{array}$ & $\begin{array}{l}10 \% \text { fluct./cycle } \\
\text { in spatial separation } \\
\text { or velocity difference }\end{array}$ & $8[0.1]$ \\
\hline $\begin{array}{l}\text { Detection laser } \\
\text { frequency noise }\end{array}$ & $\delta v=2 \mathrm{kHz}$ & 9 [0.9] \\
\hline
\end{tabular}


applied sciences. By virtue of current technological and scientific developments, this fascinating goal is now becoming closer. Q-WEP and STE-QUEST ATI aim to push current limits on the Weak Equivalence Principle by one and two orders of magnitude, respectively. They will motivate new physics if a violation of the equivalence principle is probed, or at least impose a new upper limit for possible violations. In both cases the result of the mission will help to further understand General Relativity. Additionally, no quantum test of the equivalence principle with competitive precision to state of the art test has been performed so far. Exploiting the quantum nature of the test is expected to yield additional insights into the field of General Relativity.

\section{References}

[1] G. Lamporesi, A. Bertoldi, L. Cacciapuoti, M. Prevedelli, G. M. Tino, Determination of the Newtonian Gravitational Constant Using Atom Interferometry, Phys. Rev. Lett. 100 (2008) 050801.

[2] J. B. Fixler, G. T. Foster, J. M. McGuirk, M. Kasevich, Atom Interferometer Measurement of the Newtonian Constant of Gravity, Science 315 (2007) 74.

[3] M. de Angelis, A. Bertoldi, L. Cacciapuoti, A. Giorgini, G. Lamporesi, M. Prevedelli, G. Saccorotti, F. Sorrentino, G. M. Tino, Precision gravimetry with atomic sensors, Meas. Sci. Technol. 20 (2009) 022001.

[4] A. Peters, K. Y. Chung, S. Chu, Measurement of gravitational acceleration by dropping atoms, Nature 400 (1999) 849.

[5] M. J. Snadden, J. M. McGuirk, P. Bouyer, K. G. Haritos, M. A. Kasevich, Measurement of Earth's Gravity Gradient with an Atom Interferometer-Based Gravity Gradiometer, Phys. Rev. Lett. 81 (1998) 971.

[6] F. Sorrentino, Y.-H. Lien, G. Rosi, G. M. Tino, L. Cacciapuoti, M. Prevedelli, Sensitive gravity-gradiometry with atom interferometry: progress towards an improved determination of the gravitational constant, New J. Phys. 12 (2010) 095009.

[7] S. Dimopoulos, P. Graham, J. Hogan, M. Kasevich, Testing general relativity with atom interferometer, Phys. Rev. Lett. 98 (2007) 111102.

[8] H. Müller, S.-W. Chiow, S. Herrmann, S. Chu, K.-Y. Chung, Atom-Interferometry Tests of the Isotropy of Post-Newtonian Gravity, Phys. Rev. Lett. 100 (2008) 031101.

[9] S. Dimopoulos, A. A. Geraci, Phys. Rev. D 68 (2003) 124021.

[10] F. Sorrentino, A. Alberti, G. Ferrari, V. V. Ivanov, N. Poli, M. Schioppo, G. M. Tino, Quantum sensor for atom-surface interactions below $10 \mu$ m, Phys. Rev. A 79 (2009) 013409.

[11] J. G. Williams, S. G. Turyshev, D. H. Boggs, Progress in lunar laser ranging tests of relativistic gravity, Phys. Rev. Lett. 93 (2004) 261101.

[12] S. Schlamminger, K.-Y. Choi, T. A. Wagner, J. H. Gundlach, E. G. Adelberger, Phys. Rev. Lett. 100 (2008) 041101.

[13] S. Fray, C. A. Diez, T. W. Hänsch, M. Weitz, Atomic Interferometer with Amplitude Gratings of Light and Its Applications to Atom Based Tests of the Equivalence Principles, Phys. Rev. Lett. 93 (2004) 240404.

[14] F. Sorrentino, K. Bongs, P. Bouyer, L. Cacciapuoti, M. de Angelis, H. Dittus, W. Ertmer, M. Hauth, S. Herrmann, M. Inguscio, E. Kajari, T. Könemann, C. L. A. L. G. Modugno, F. P. dos Santos, A. Peters, M. Prevedelli, E. M. Rasel, W. P. S. M. Schmidt,
A. Senger, K. S. and. G. Stern, G. M. Tino, R. Walser, A compact atom interferometer for future space missions, Microgravity Sci. Tech. J. 22 (2010) 551.

[15] G. S. et al., Light pulse atom interferometry in microgravity, Eur. Phys. J. D 53 (2009) 353.

[16] e. a. T. van Zoest, Bose-einstein condensation in microgravity, Science 328 (2010) 1540.

[17] H. M. et al., Interferometry with bose-einstein condensates in microgravity, Phys. Rev. Lett. 110 (2013) 093602.

[18] Atom interferometry test of the weak equivalence principle in space, statement of work, appendix 1 to esa itt ao/1$6763 / 11 / \mathrm{nl} / \mathrm{af}$.

[19] T. Lévéque, A. Gauguet, F. Michaud, F. P. D. Santos, A. Landragin, Enhancing the area of a raman atom interferometer using a versatile double-diffraction technique, Phys. Rev. Lett. 103 (2009) 080405.

[20] G. T. Foster, J. B. Fixler, J. M. McGuirk, M. A. Kasevich, Novel Method of Phase Extraction Between Coupled Atom Interferometers Using Ellipse-Specific Fitting, Opt. Lett. 27 (2002) 951.

[21] F. Sorrentino, A. Bertoldi, Q. Bodart, L. Cacciapuoti, M. de Angelis, Y. Lien, M. Prevedelli, G. Rosi, G. Tino, Simultaneous measurement of gravity acceleration and gravity gradient with an atom interferometer, Applied Physics Letters 101 (11) (2012) 114106-114106.

[22] P. Cheinet, B. Canuel, F. P. D. Santos, A. Gauguet, F. YverLeduc, A. Landragin, Measurement of the sensitivity function in a time-domain atomic interferometer, IEEE Trans. on Instr. and Meas. 57 (2008) 1141.

[23] J. M. Hogan, D. M. S. Johnson, M. A. Kasevich, Light-pulse atom interferometry, in: E. Arimondo, W. Ertmer, W. P. Schleich, E. Rasel (Eds.), Atom Optics and Space Physics, Proceedings of the International School of Physics "Enrico Fermi" ; course 168, IOS Press Amsterdam, Washington, DC, 2009, p. 411.

[24] P.-C. K. S.-Y. Lan and, B. Estey, P. Haslinger, H. Müller, Influence of the coriolis force in atom interferometry, Phys. Rev. Lett. 108 (2012) 090402.

[25] A. Louchet-Chauvet, T. Farah, Q. Bodart, A. Clairon, A. Landragin, S. Merlet, F. P. D. Santos, The influence of transverse motion within an atomic gravimeter, New J. Phys. 13 (2011) 065025.

[26] J. E. Debs, P. A. Altin, T. H. Barter, D. Döring, G. R. Dennis, G. McDonald, R. P. Anderson, J. D. Close, N. P. Robins, Coldatom gravimetry with a bose-einstein condensate, Phys. Rev. A 84 (2011) 033610. 